\title{
A Research of High-performance Geometric Precision Correction Based on Reference Image Database
}

\author{
Zhao Lingjun $^{1,2, a}$ Zhang Wanfeng ${ }^{1,2, b}$ Liu Dingsheng $^{1, c}$ \\ ${ }^{1}$ Center for Earth Observation and Digital Earth,CAS, Beijing,China \\ ${ }^{2}$ University of Chinese Academy of Sciences,Beijing,China \\ `ljzhao@ceode.ac.cn, ’bwfzhang@ceode.ac.cn, dsliu@ceode.ac.cn
}

\begin{abstract}
Keywords: Geometric Precision Correction; Reference Image Database; Parallel; High Performance
\end{abstract}

\begin{abstract}
Geometric precision correction of remote sensing image plays a more and more important role in the application of high precision remote sensing. A reference image database is built up based on PostgreSQL, an open-source relation database, and ArcSDE, a widely used spatial data engine. Compared with single reference image, the reference image database has the advantages of managing convenience, lower-cost maintenance and high retrieval efficiency. Meanwhile, the correction accuracy is boasted by Gopfert algorithm , and efficiency is improved by advanced parallel model based on parallel I/O and I/O hidden strategy. Beyond this, combined with MapServer which can rapidly display images, a geometric precision correction system of high performance with dual servers is formed, with which mass production ability is achieved.
\end{abstract}

\section{Introduction}

Today, remote sensing technology plays an more and more important role in the fields of city planning, resources development, environmental protection, marine monitoring and etc, while the demands to the remote sensing from all the fields are corresponding increased. The precision of L2 geometrical products corrected by the satellite parameters cann't suit the high- precision application, so it becomes a very meaningful work to make a geometric precision correction based on L2 products used GCPs(ground control points) ${ }^{[1]}$.

As the most important premise, the selection of GCPs should obey the principle of accurate location and not change with time change. The obtained methods are mainly three methods ,manual inputing, from single reference image and from reference image database. Theeference image database has advantage of Low maintenance, good portabilityefficient, easy to operate and so on, which is that the manual inputing and single reference image can not possess.

As a widely used data source, remote sensing image has the characteristices of short obtained period, more remote sensing platforms and huge data size, so it can not suit the process of huge data which is more than 1TB that simply depends on updating the computer hardware. Especially, the large amount of computation of resample in geometric precision correction place greater demands on computers, so parallel process should be drawn into which can process huge data well.

Based on the above considerations, this paper divides the geometric precision correction into two parts to research, one is to study how to build efficient reference image database and select GCPs easily, and the other is to adopt suitable correction model and parallel strategy to enhance the correction precision and performance by the GCPs. Based on the two key technologies above, an efficient and, convenient software is built, which can fulfill the requirement of mass mass production.

\section{Reference Image Database}

The main two factor of reference image database are relation database and spatial data engine. The common databases are Oracle, SQLServer, PostgreSQL and etc ${ }^{[2]}$, while the spatial data engine are ArcSDE, Supermap SDX+, Spatial Wrae, Oracle Spatial and so on. This paper adopted the open-source PostgreSQL and a widly-used ArcSDE. 
PostgreSQL and ArcSDE. PostgreSQL is an object-relational database management system (ORDBMS) based on POSTGRES, Version 4.2, developed at the University of California at Berkeley Computer Science Department. POSTGRES pioneered many concepts that only became available in some commercial database systems much later. It supports a large part of the SQL standard and offers many modern features such as complex queries ,foreign keys, triggers, views ,transactional integrity and multiversion concurrency control ${ }^{[3]}$. it support sof many mainstream operatingsystem, such as Windows, Linux, Mac OS X,Solaris and etc, which lays the foundation on business development and scientific research.

ArcSDE is the GIS server software developed by ERSI, which is used to build server and web GIS application. It is a bridge between ArcGIS and the relation database, in other word, it is the spatial data engine of ArcGIS, by which RDBMS can manage the spatial data. The architecture of ArcSDE is $\mathrm{C} / \mathrm{S}$, so many users can concurrently visit and operate the same database through ArcSDE. ArcSDE supports not only business databases such as Oracle, SQL Server, IBM DB2, but also the open-source PostgreSQL on its 9.3 version.

Build reference image database. The reference image database plays an important role in Geometric precision correction.and there are three main steps to build it by arcsde and postgreSQL, which are listed as following.

1) To build image pyramid

The reference images have the features of wide scope and huge data size, which brings huge burden to network transmission and CPU process when it zooms under one single scale. And image pyramid can solve the above problems effectively. Under a certain standard, we build a serial of image layer from original data, and each layer reflects the information of different scale. This paper realizes the image pyramid by GDAL.

2) To Load reference image to database

ST_Geometry type is provide by the ArcSDE for PostgreSQL to storage Geometry Data, which follows the standard of ISO and OGC, and can be directly fetched by SQL. Corresponding SDE schema must be built firstly before storaging spatial data by ST_Geometry. We can use the client tool provide by ArcSDE to load the reference image whose image pyramid is already built to the PostgreSQL Server after starting the ArcSDE Server deployed on the server.

3) To Build index table

To accurately and rapidly find the reference image in need, index table is built for all the reference images. Index information includes the geometrical sclope, lines, rows, projection string, EPSG code and other information for each reference image. We use Jave to operate the PostgreSQL database to implement the table.

\section{Parallel Geometric Precision Correction}

Polygon model is always adopted in geometric precision correction, but we use the Gopfert model which can get higher accurary. At the same time, some advanced parallel strategies are used in the interpolation step which is the most time-consuming, to shorten the process time.

Gopfert model. Gopfert is a flow of geometric precision correction with high-accuracy, which combines the polygon model and multi-quadric functions model.

Gopfert algorithm is composed of four principal steps, among which $(\mathrm{u}, \mathrm{v})$ is the postion of one pixel in the original image, and $(x, y)$ is the corresponding position in the result image,

1) Model the global geometric distortion of the $(x, y)=f(u, v)$ using a bivariate mapping polynomial expression. This is an inverse transformation intended to remove the global trend in the data.

$[\mathrm{x}, \mathrm{y}] \mathrm{p}=[\mathrm{Px}(\mathrm{u}, \mathrm{v}, \mathrm{x}), \mathrm{Py}(\mathrm{u}, \mathrm{v}, \mathrm{y})]$

2) Find the residual distortion (actual - predicted) from [1].

$\left(x^{r}, y^{r}\right)=[x, y]^{R}=\left[[x, y]-[x, y]^{p}\right]$ 
3) Model the residual distortion (not to be confused with error) from [2] using the MQ method. That is, model the distortion as $(\mathrm{xr}, \mathrm{yr})=\mathrm{f}(\mathrm{u}, \mathrm{v})$

$[\mathrm{x}, \mathrm{y}]^{\mathrm{MQ}}=\left[\mathrm{MQx}\left(\mathrm{u}, \mathrm{v}, \mathrm{x}^{\mathrm{r}}\right), \mathrm{MQy}\left(\mathrm{u}, \mathrm{v}, \mathrm{y}^{\mathrm{r}}\right)\right]$

4) Add the predicted values from [1] and the MQ values from [3] to generate (x,y) as a function of $(\mathrm{u}, \mathrm{v})$.

$(\mathrm{x}, \mathrm{y})=[\mathrm{x}, \mathrm{y}]=[\mathrm{x}, \mathrm{y}]^{\mathrm{p}}+[\mathrm{x}, \mathrm{y}]^{\mathrm{MQ}}$

5) Gray Value Interpolation with the nearest-neighbor method, the bilinear method or the cubic convolution method ${ }^{[4]}$.

The effective parallelization of geometric precision correction. From above, resample is the most time-consuming step for its huge comupute, and Parallelization is an effective way to speed up the Remote Sensing image processing ${ }^{[5]}$ Based on traditional Master-Slave parallel processing model ${ }^{[6]}$, A parallel I/O and I/O hidden strategy is adopted in this paper, which is very effective, and the complete parallel extraction flow is showed in the Figure below.

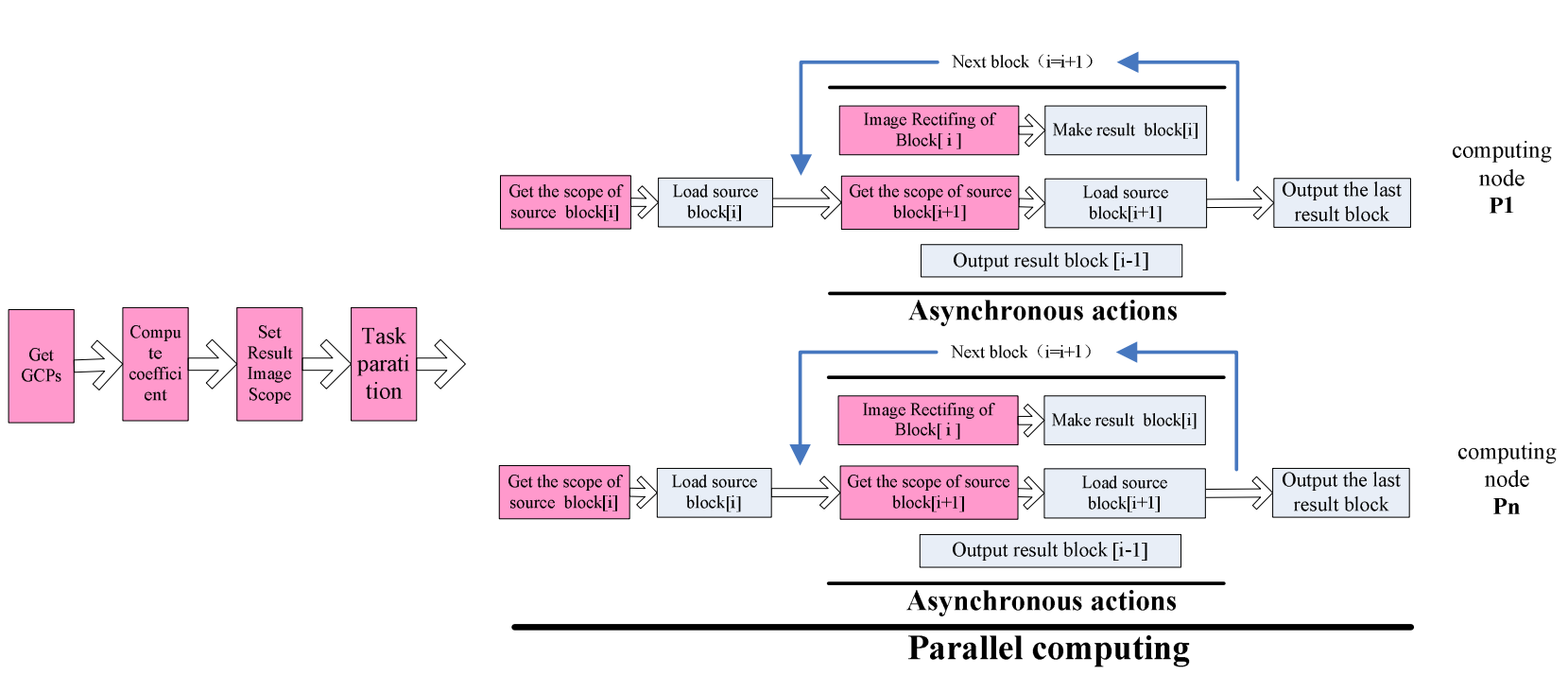

Fig. 1 The parallel recification of images

Master node (manipulating the whole mission):

1) To get gcps and compute coefficients;

2) To set up the result image scope;

3) To divide missions.

4) According to the number of computing nodes and given policy, master node divides the whole result image into result blocks averagely, considering the boundary redundancy among blocks to ensure the continuity of the result image. It divides the whole mission into sub-missions which are assigned to computing nodes respectively and are related to the result blocks one-to-one.

Computing nodes (parallel performance):

1) To get the first result block which corresponds to the source block[1] in source image;

2) To load the source block[1] into the EMS memory concurrently by parallel file system according to the scope of source block[1];

3) To execute a) c) concurrently and asynchronously, the initial value of $i$ is 1 ,

4) to recitify the current source block[i] to get the result block[i];

5) Meanwhile, by using multithreading technology to derive a thread to calculate the scope of source block $[i+1]$ corresponding to the next result block. And then to read it asynchronously by using asynchronous operation of MPI-I/O based on parallel file system PVFS2;.

6) At the same time, if the former result block[i-1] is not output, the computing node outputs it asynchronously by using asynchronous operation of MPI-I/O based on PVSF2; 
7) Check whether the Asynchronous operation of source block [i+1] has completed or not, if some blocks haven't completed the water area extraction, then repeat step 3and 4, otherwise continue to execute step 5;

8) To output the last result block[last].

\section{High-performace Geometric Precision Correction System}

Geometric precision correction touches on two important parts, GCPs selection and Correction, so it is utmost importance to design suitable software architecture. A image server based on MapServer is made, which realizes the human interaction of images between server and client. And also, another OMS server is made to dispatch and execute the correction tasks.

MapServer and image display. MapServer is a popular open source project whose purpose is to display dynamic spatial maps over the Internet and can run on various operating systems (Windows, Linux, Mac OS X, etc.). MapServer is a CGI program, and can be extended and customized through MapScript or templating, and MapScript currently exists in PHP, Perl, Python, Ruby, Tcl, Java, and .NET flavors ${ }^{[7]}$.

MapServer is developed by $\mathrm{C}$ language and flows s of network standardizations drew up by OGC,such as WMS, WFS, WCS,WMS, GML and etc. It supports any vector and raster input data formats by GEOS, GDAL and OGR, and also supports many geometric projection by Proj.4 libaray. MapServer integrates PostGIS ,a open-source spatial data engine, and PostgreSQL, a open-source database, to realize the storage and quick find of the spatial data. Meanwhile, it selectively supports the ArcSDE, a business software, which make its geographic structure more perfect.

The IE browser can display the processed image if we create the Map file under a certain standard and visit the MapServer by http protocol. The MapServer resamples the original image by the geometric scope and other continuity constrains that obtained from the url and make a temporary picture to transfer and display. The temporary picture is always small, so it can be displayed quickly. The structure of high-performace geometric precision correction system. In terms of Convenience and efficiency, dual servers structure is adopted to build geometric precision correction system of high performance based on reference image database, and the software architecture diagram is showed as below.

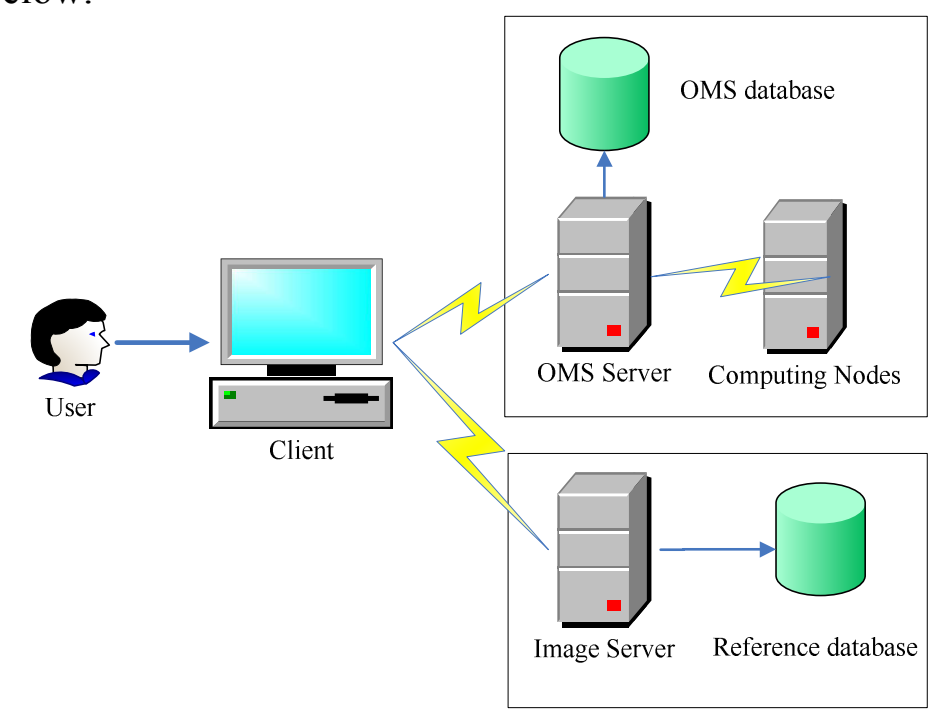

Fig. 2 Software architecture

The image server is responsible for interaction, which display the image on-demand by MapServer, and the HTTP protocol is adopted between the image server and client. The OMS server is due to dispatch and execute the tasks. According to the users' demands, the OMS server launches the correction task, and parellelly process the tasks in the computing nodes with the GCP files that are uploaded by the client. Between the OMS server and client, the web service protocol is used. 
The flow of high-performance geometric precision correction. The total flow of high-performance remote sensing image geometric precision correction based on reference image database is showed as below.

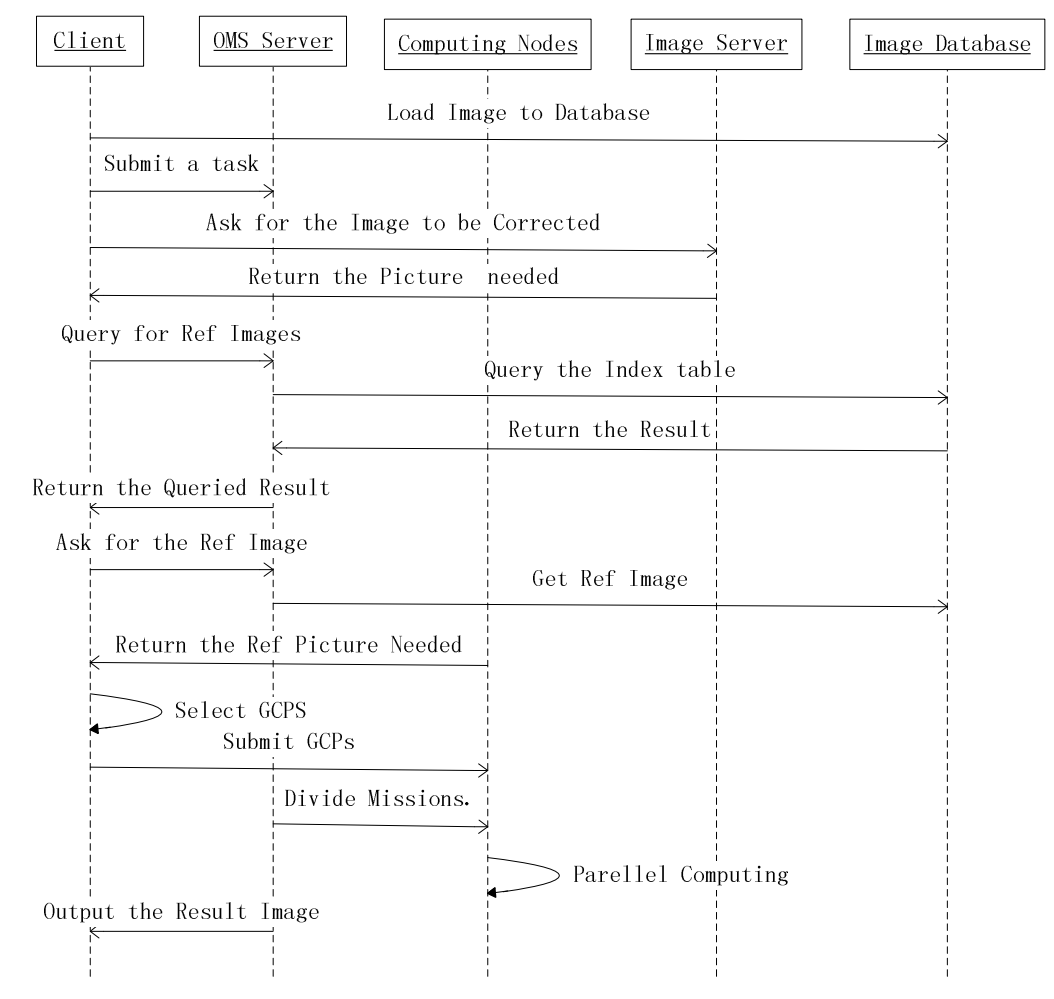

Fig. 3 The total flow

1) The system storage the reference images to PostgreSQL database by ArcSDE .

2) The client select the image to be corrected and ask for the image server to display. MapServer on the image server accepts the parameters form the Apache, find the image through the data source and connection type that defined in the mapfile which can get from the parameters, and return the process result of the image to the client to display.

3) Through the geography scope and the web service interface provided by the OMS server,the client find the ref image name from the PostgreSQL database and ask for the Image server to display.

4) The client select GCPS Interactively, make a GCP file, and upload to the OMS server, the OMS execute the correction task by GCP file and the image to be corrected.

\section{Experiment and Summary}

Selecting a multispectral image from Beijing NO.1 satellite as the test data, whose geographical range is $253 \mathrm{~km} \times 225 \mathrm{~km}$ and resolution is $32 \mathrm{~m} \times 32 \mathrm{~m}$, and selecting a landsat- 7 orthorectification image as the reference image, our precision test indicates that the correction errors of Gopfert model are mostly less than 2 pixels, which is much better than the traditional ones in accuracy. And also,the parallelization efficiency of new method always kept better than $90 \%$, which is obviously superior to the traditional one.

In conclusion, a geometric precision correction system of high performance with dual servers is formed. In the system, some advanced technologies are adopted. PostgreSQL and ArcSDE are used to build reference image database, MapServer is applied to quickly display images and select GCPs, Gopfert model is adopted to enhance correction accuracy, parallel I/O and I/O hidden strategy are implemented to enhance the correction efficiency. And this system is adopted by some 863 projects such as Coastal zone remote sensing. The practices shows, this system has the advantages of efficient,high- accuracy and easy to operate, and can fulfill the requirement of mass mass production. 


\section{References}

[1] Barbara Zitova, Jan Flusser,.Image registration methods: a survey. Image and Vision Computing. 21(2003),977-1000.

[2] WANG Ming-chong, ZHAO Jun,LI Yu-lin,Analysis of Spatial Database Engine and Its Solutions. Geomatric World. 4(2006) 36-66

[3] Information on http://www.postgresql.org/docs/8.3/static/intro-whatis.html

[4] YANG Gao-pan, GUO Shi-hong, IKEN Lei-ping, Research on Correction of Geometric in High-resolution Satellite Image,Computer Knowledge and Technology.6(2010)2235-2237.

[5] Zhao Lingjun, Liu Ding-sheng, Li Guo-qing, Zhang Wen-yi, Study of High-Performance Precision Correction for Satellite Image, Remote Sensing For Land \& Resouces. 71(2007), P49-52.

[6] Warpenburg, M.R. and Siegel, L.J.,SIMD image resampling,IEEE Transactions on Computers, 31(1982)934-942

[7] Information on http://MapServer.org/introduction.html 